\title{
Globe
}

Revue internationale d'études québécoises

\section{Lecture du débat sur les accommodements raisonnables}

\section{Micheline Labelle et Jean-Claude Icart}

Volume 10, numéro 1, 2007

URI : https://id.erudit.org/iderudit/1000082ar

DOI : https://doi.org/10.7202/1000082ar

Aller au sommaire du numéro

Éditeur(s)

Globe, Revue internationale d'études québécoises

ISSN

1481-5869 (imprimé)

1923-8231 (numérique)

Découvrir la revue

Citer cet article

Labelle, M. \& Icart, J.-C. (2007). Lecture du débat sur les accommodements raisonnables. Globe, 10(1), 121-136. https://doi.org/10.7202/1000082ar d'utilisation que vous pouvez consulter en ligne.

https://apropos.erudit.org/fr/usagers/politique-dutilisation/ 


\title{
PERSPECTIVE
}

\section{LECTURE DU DÉBAT SUR LES ACCOMMODEMENTS RAISONNABLES}

\author{
MICHELINE LABELLE \\ Département de sociologie, \\ Centre de recherche sur l'ummigratıon, l'ethnicité et la citoyenneté \\ Université du Québec à Montréal \\ JEAN-CLAUDE ICART \\ Observatoire international sur le racisme et les discnminations (CRIEC) \\ Université du Québec à Montréal
}

Le 26 mars 2007, le Québec a été frappé par un véritable séisme électoral, se retrouvant avec un gouvernement minoritaire pour la première fois depuis plus d'un siècle, depuis 1878 plus précisément. Il s'est également découvert morcelé en trois segments bien distincts, représenté chacun à l'Assemblée nationale par une formation politique différente, ce qui a soulevé des inquiétudes chez bien des observateurs. La grande surprise a été la progression de l'ADQ (Action démocratique du Québec), une formation politique de droite considérée jusque-là comme marginale et qui constitue aujourd'hui l'opposition officielle. Ce bouleversement est dû en partie au débat sur les accommodements raisonnables, qui a occupé une grande place dans l'espace médiatique et l'opinion publique au cours de la dernière année, et à la position claire de Mario Dumont sur la question. Comme l'écrit Éric Bédard: "C'est qu'aujourd'hui, le débat sur les accommodements 
raisonnables a la même portée symbolique et politique que le débat sur la langue au tournant des années $70^{1} . "$

En effet, les revendications issues de diverses minorités religieuses, ainsi qu'une critique ouverte des excès attribués au multiculturalisme, n'ont cessé d'alimenter les controverses. Or, il existe une grande confusion autour du multiculturalisme. Ce concept peut signifier une philosophie politique de remise en question des visions classiques de l'État-nation, un discours postcolonialiste, un ensemble de dispositifs politiques et institutionnels qui mettent l'accent sur la justice et la reconnaissance de la dignité des acteurs sociaux, une politique publique comme la politique fédérale canadienne ou australienne, une simple réalité sociodémographique, etc. Les interrogations préexistantes sur la portée des chartes québécoise et canadienne des droits et libertés de la personne, sur la laïcité, de même que sur l'identité québécoise se sont alimentées, en partie, à même un courant idéologique néoconservateur s'affichant désormais ouvertement.

La première partie de cet article relate quelques-uns des récents moments forts du débat sur les accommodements raisonnables et le racisme au Québec. Une deuxième partie s'attache à des enjeux sociétaux plus larges et aux facteurs internationaux et nationaux qui ont exercé une influence structurante sur ce débat.

\section{UNE ANNÉE MOUVEMENTÉE. MARS 2006-MARS 2007}

D'entrée de jeu, rappelons que la Commission des droits de la personne et des droits de la jeunesse du Québec (CDPDJ) utilise le concept d'accommodement raisonnable dans un sens précis:

Obligation juridique découlant du droit à l'égalité, applicable dans une situation de discrimination, et consistant à aménager une norme ou une pratique de portée universelle, en accordant un traitement différentiel à une personne qui, autrement, serait pénalisée par l'application d'une telle norme. Il n'y a pas d'obligation d'accommodement en cas de contrainte excessive ${ }^{2}$.

L'accommodement raisonnable découle de deux concepts juridiques: l'égalité et la discrimination. Au milieu des années 1980, il fait son apparition dans le domaine du droit québécois et canadien au sein des lois et

$$
+4
$$

1. Érı BÉDARD, aLa colère "bleue" des nationalisces", Le Devoir, 26 avril 2007, p. A7.

2. Marc-André DowD, Accommodements raisonnables. Eviter les derapages, Montréal, CDPDJ, novembre 2006 (Cat. 2.600.222). 
chartes dites "quasi constitutionnelles», qui garantissent le droit à l'égalité et interdisent en conséquence les pratiques discriminatoires ${ }^{3}$. Il s'agit donc essentiellement de remédier à des situations de discrimination, idéalement dans le cadre d'une entente négociée, par l'adaptation de pratiques ou de politiques d'une institution, et ce, dans les limites du raisonnable.

La moitié des accommodements raisonnables concernent les personnes souffrant d'un handicap: permettre à un non-voyant de voter, à un employé handicapé de travailler, etc. Les autres demandes sont présentées par des Québécois de troisième ou de quatrième génération convertis à de nouveaux mouvements religieux, par des témoins de Jéhovah, des protestants, des Juifs hassidiques, etc. Une minorité de demandes concernent les nouveaux immigrants. Cela permet de voir que l'accommodement raisonnable n'est ni une problématique d'immigration ni une problématique de multiculturalisme (au sens de la politique fédérale du multiculturalisme). Or, elle a été traitée comme relevant directement de ces dernières.

\section{DES MOMENTS CRITIQUES}

Le 2 mars 2006, la Cour suprême du Canada décidait à l'unanimité que l'interdiction du port d'un poignard de cérémonie, le kirpan, établie par le Conseil des commissaires de la Commission scolaire Marguerite-Bourgeoys, violait la liberté de religion garantie par la Charte canadienne des droits et libertés.

Cette affaire avait commencé en décembre 2001, quand la Commission scolaire Marguerite-Bourgeoys avait conclu avec une famille sikh orthodoxe une entente permettant à leur enfant de porter son kirpan à l'école, à condition de le sceller à l'intérieur de ses vêtements. Deux mois plus tard, le Conseil des commissaires de la commission scolaire refusait d'entériner l'entente, car elle contrevenait au code de conduite de l'école, qui prohibe le port d'armes et d'objets dangereux. La Cour supérieure du Québec accueillit favorablement la requête de la famille en jugement déclaratoire, décision qui fut cassée par la Cour d'appel du Québec. La famille s'est alors pourvue devant la Cour suprême du Canada, qui a estimé que la liberté de religion était en jeu puisque la croyance religieuse atteinte était sincère:

$$
++*
$$

3. Pierre BOSSET, „Les fondements juridiques er l'évolution de l'obligation d'accommodement raisonnable», Myriam JEZZQUEL [Ed.], Les accommodements raisonnables. Quoi, comment, jusqu'où?, Cowansville, Éditions Yvon Blais, 2007, p. 7-27. 
Le fait que plusieurs personnes pratiquent la même religion de façon différente n'invalide pas pour autant la demande de celui qui allègue une violation à sa liberté de religion. Ce qui importe, c'est que cette personne démontre qu'elle croit sincèrement que sa religion lui impose une certaine croyance ou pratique. La croyance religieuse invoquée doit être avancée de bonne foi, elle ne doit pas être fictive, ni arbitraire, et elle ne doit pas constituer un artifice ${ }^{4}$.

La Cour suprême a également estimé que les effets préjudiciables de l'interdiction totale surpasseraient ses effets bénéfiques. Cette décision provoqua un véritable tollé: les tribunes téléphoniques et les courriers des lecteurs des grands quotidiens furent littéralement pris d'assaut.

Le 22 mars 2006, la CDPDJ se prononçait sur une plainte déposée par 113 étudiants musulmans contre l'École de technologie supérieure (ETS), membre du réseau de l'Université du Québec. Ces derniers soutenaient ne pouvoir effectuer les cinq prières quotidiennes obligatoires dans le cadre de leur religion, l'ÉTS ayant refusé de leur accorder un lieu de prière au nom du caractère laïque de l'établissement. La CDPDJ a conclu que l'ÉTS avait une obligation d'accommodement raisonnable à l'égard de ces étudiants afin qu'ils puissent prier sur une base régulière, selon des conditions qui respectent leur droit à la sauvegarde de leur dignité sans toutefois imposer un local spécifique pour une religion donnée. D’autres griefs formulés par les étudiants furent toutefois rejetés.

$\mathrm{La}$ CDPDJ reconnaissait cependant que "la réponse au cas par cas" n'était pas satisfaisante et annonçait la tenue d'une consultation sur les limites et la portée des accommodements raisonnables, sur la façon de "concilier un idéal de laïcité avec le respect des droits religieux" nisme n'amorça cette consultation qu'en janvier 2007.

En juillet 2006, devant l'accroissement des demandes concernant le sexe des médecins et la multiplication des incidents dans divers hôpitaux de Montréal, le Centre hospitalier de l'Université de Montréal décida de "prendre le problème de front" et d'accueillir les femmes enceintes «avec une déclaration écrite spécifiant qu'il est impossible de garantir que le médecin du sexe de leur choix soit présent à leur accouchement ${ }^{6} »$.

$$
++
$$

4. Mulani c. Commission scolaire Marguerite-Bourgeoys, 2006 CSC 6, [2006] 1 R.C.S. 256.

5. Marc-André DOWD, cıté par Clairandrée CAUCHY, "L'ÉTS doit offrir un lieu de prière aux érudianss musulmans", Le Devort, 23 mars 2006, hrtp://www.ledevoir.com/2006/03/23/104978.html (31 juillet 2007).

6. Agnes GRUDA, *Santé. Jusqu'où aller? ", La Presse, 24 septembre 2006, http://www.cyberpresse.ca/arricle/20060924/ ACTUALITES/60924062 (26 avrl 2007). 
Le 11 octobre 2006, le ministre de l'Éducation, du Loisir et du Sport du Québec annonçait la mise sur pied d'un Comité consultatif sur l'intégration et l'accommodement raisonnable en milieu scolaire, chargé de faire le point sur "l'intégration, dans le réseau de l'éducation, des jeunes issus de communautés culturelles, religieuses et linguistiques différentes ainsi que sur la gestion de la diversité au moyen d'accommodements raisonnables»:

Le Comité consultatif sur l'intégration et l'accommodement raisonnable aura donc pour mandat de proposer une stratégie et des outils pertinents afin d'aider le ministère de l'Éducation, du Loisir et du Sport ainsi que les membres du réseau de l'éducation à cheminer sur la question de l'intégration et de la gestion de la diversité culturelle et religieuse. Il devra produire une définition claire et accessible de ce qu'est un accommodement raisonnable. Il devra également produire un bilan des initiatives dans le domaine et documenter les nouveaux enjeux relatifs à l'adaptation de l'école québécoise à la diversité ethnoculturelle, religieuse et linguistique. Il dressera l'inventaire des outils d'information et de formation accessibles aux différentes catégories de personnel scolaire et il proposera, enfin, la production de documents et d'outils à l'intention du réseau de l'éducation'.

Les craintes liées aux accommodements raisonnables s'exprimèrent de plus en plus lorsque, le 25 janvier 2007, Hérouxville, un petit village de Mauricie de 1300 habitants, décida de s'attaquer à cette question en adoptant des "normes de vie ${ }^{8}$ " qui firent grand bruit à l'échelle nationale. Ce "code de conduite pour les immigrants" contenait dans sa version originale à la fois un énoncé de principe contre les discriminations et un condensé des pires clichés concernant les musulmans. Il reçut une importante couverture médiatique à travers le monde entier. Originaire de la Dominique, le seul immigrant de cette localité de 1300 personnes en a fait l'analyse suivante:

Les conseillers municipaux ont voulu tirer une sonnette d'alarme [...]. Ils l'ont fait maladroitement, mais ils soulignent une préoccupation que nous avons tous. Le multiculturalisme à la canadienne est une illusion. Il ne faut pas parler de réussite quand on

$$
\div \div+
$$

7. Marie-Claude LAVIGNE, «Le ministre Jean-Marc Fournier annonce la création d'un Comicé consulcatif sur l'intégration et l'accommodernent raisonnable en milieu scolairew, communiqué de presse, ministère de l'Éducation, du Loisir et du Sport, gouvernement du Quebec, 11 octobre 2006, http://www.mels.gouv.qc.ca/CPRESS/cprss2006/ c061011.asp (31 juillet 2007).

8. Municipalité de Hérouxville, *Normes de vien, hatp://municipalite.herouxville.qc.ca/normes.pdf (31 juillet 2007). 
assiste à un non-mélange et à la mise côte à côte de différentes cultures?.

Ce code de conduite traduisait effectivement l'inquiétude de plusieurs municipalités par rapport aux changements démographiques et culturels observés surtout dans la grande région métropolitaine de Montréal, et par rapport aux effets pervers attribués à la reconnaissance de la diversité. Il témoignait aussi d'une certaine animosité relative à la perception de la "domination de Montréal» sur les régions du Québec.

Dans un tel contexte, le 8 février 2007, le premier ministre du Québec annonçait la création de la Commission de consultation sur les pratiques d'accommodements reliées aux différences culturelles. Cette commission, coprésidée par le sociologue Gérard Bouchard et le philosophe Charles Taylor, a pour mandat principal de formuler des recommandations au gouvernement afin que les pratiques d'accommodements soient respectueuses des valeurs communes des Québécois.

\section{LE RÔLE DES MÉDIAS}

Les médias jouèrent un rôle de premier plan dans l'évolution du débat public sur les accommodements raisonnables dès l'automne 2006. En septembre 2006, un sondage commandé par les journaux La Presse et Toronto Star révéla que les Québécois étaient plus réticents que l'ensemble des Canadiens quant au port de signes religieux dans l'espace public ${ }^{10}$. Peu après l'annonce de la mise sur pied du Comité consultatif sur l'intégration et l'accommodement raisonnable en milieu scolaire, une série de révélations plus sensationnalistes les unes que les autres fit déraper le débat avant qu'il n'ait pu véritablement commencer.

Ainsi, durant la première quinzaine de novembre 2006, on apprenait notamment, coup sur coup, que plusieurs actions contestables et contestées, présentées faussement comme des "accommodements raisonnables", étaient survenues: la direction d'un centre sportif du YMCA de Montréal avait installé, à la demande de Juifs hassidiques d'Outremont, quatre fenêtres givrées afin d'éviter que les jeunes garçons fréquentant leur synagogue ne voient les femmes à l'entraînement; dans un article du bulletin

$$
4+4
$$

9. Emmanuelle LANGLoIS, "La croisade xénophobe d'un village québécois", Lzbératzon, 16 février 2007. "La croisade xénophobe d'un village québécois n, Liberation, 16 février 2007. http://www.liberation.fr/actia;ote/ monde/2345634.FR.php (24 avril 2007).

10. Kacia GaGNON et Agnès GRUDA, "Les Quebecous plus récicents que l'ensemble des Canadiens aux signes relıgieux", La Presse, 22 septembre 2006, http://www.vigile.net /article2087.html (31 juillet 2007). 
interne du Service de police de Montréal (rubrique "Trucs du métier»), un inspecteur avait recommandé à ses collègues féminines de céder leur place à un collègue masculin lors d'interventions auprès des Juifs hassidiques; un Centre local de services communautaires (CLSC) de Montréal aurait refusé que les hommes assistent à des cours prénataux pour accommoder les femmes musulmanes, hindoues ou sikhs, etc.

Bref, la presse semblait se livrer à une véritable chasse aux arrangements les plus déraisonnables possible. Dans la foulée, tout accord pouvant être associé de près ou de loin à des pratiques de bonne entente et à la gestion au quotidien de la diversité culturelle ou religieuse fut qualifié $\mathrm{d}$ ' "accommodement raisonnable", ce qui vida le terme de son contenu et ouvrit la porte à toutes sortes de glissements.

Les résultats d'un autre sondage réalisé par le consortium SOMLa Presse-Le Soleil furent diffusés le 29 décembre 2006. Ils montraient que près de six Québécois sur dix $(58,6 \%)$ jugeaient la société trop tolérante en matière d'accommodements raisonnables, ce pourcentage augmentant en fonction du revenu et du niveau de scolarité, alors que l'opinion des jeunes répondants allait à contre-courant. Par ailleurs, une bonne majorité $(68,1 \%)$ considérait que l'immigration est un atout pour la société québécoise, et $92,2 \%$ des répondants estimaient que le Québec est "très ou assez" accueillant pour les immigrants ${ }^{11}$.

C'est cependant un sondage de la firme Léger Marketing portant sur la tolérance au Québec qui mit véritablement le feu aux poudres. Le Journal de Montréal commença la publication de l'étude par un titre-choc présenté à la une: "59\% des Québécois se disent racistes", en se basant sur une définition bancale du racisme. Ce sondage, objet de nombreuses critiques sur le plan méthodologique ${ }^{12}$, fut réalisé pour le Journal de Montréal la station de télévision TVA et la station de radio 98,5, trois médias appartenant au groupe Quebecor. Cette convergence fut mise à profit. Le sondage fit la une du Journal de Montréal durant toute la semaine et fur largement couvert par les autres médias du groupe. TVA lui consacra une émission spéciale de deux heures. L'opinion publique fut fortement ébranlée

$$
+4
$$

11. André DUCHESNE, "Des accommodements raisonnables qui incommodent", La Presse, 29 décembre 2006, http:/www.cyberpresse.ca/article/20061229/CPACTUALITES/61229001 (31 juillet 2007).

12. Rachad ANTONIUS, Jean-Claude ICART et Micheline LABELLE, "Un sondage imprudent " La Presse, 19 janvier 2007, http://www.cyberpresse.ca/article/20070119/CPOPINIONS/701 190841\&SearchID $=73269681601091$ (31 juillet 2007). Voir aussi Alexandre SHIELDS, “Tempête "identıcaire” au Québec. Charest affirme que "les Québécois ne sont pas racistes", Dumont rejette le "vieux réflexe minoritaire" ", Le Devour, 7 janvier 2007, p. A1; et Jean-Claude ICART et Micheline LABELLE, "Tolérance, racisme et sondages*, Ethaque publique, vol. 9, $n^{\circ} 1$, «L'aménagement de la diversité culturelle et religieuse», juin 2007, p. 181-186. 
par les titres-chocs et la banalisation du racisme, placé en exergue de la question des accommodements raisonnables présentés par le sondeur comme des mesures allant contre la loi et l'ordre public. Ce dossier monopolisa complètement l'espace public et alimenta une véritable guerre entre différents groupes de presse ${ }^{13}$.

Le plus grand pouvoir des médias réside dans leur capacité à focaliser l'opinion publique sur un thème, qui devient alors un enjeu de premier plan. Ce pouvoir opère par l'utilisation de différents procédés: la sélection et le traitement des informations, bien sûr, mais aussi le recours aux sondages ou, du moins, à certains types de sondages. La concurrence contribue grandement à faire des médias «des entreprises commercialement intéressées à intéresser le public à la chose publique ${ }^{14}$ ». Et la concentration médiatique que connaît le Québec aiguillonne cette concurrence. Or, tout ceci se passait en pleine période prélectorale. Ce dossier contribua donc grandement à déterminer les attentes des électeurs.

\section{LE TRAITEMENT PAR LE POLITIQUE}

Durant la première quinzaine de novembre 2006, au cours de la recherche effrénée d'arrangements déraisonnables par les médias, il était devenu clair aux yeux de certains analystes qu'un leader politique allait tôt ou tard se saisir de ce dossier et en faire son cheval de bataille. Effectivement, l'ADQ, un parti relativement marginal depuis une dizaine d'années ( 5 députés parmi les 125 élus de l'Assemblée nationale à ce moment-là), en fit le thème central de son congrès les 17 et 18 novembre 2006, à Trois-Rivières (non loin d'Hérouxville).

Pour ce parti politique, le dossier ne pouvait mieux tomber. Les dirigeants martelèrent ce thème tout au long du congrès, reprenant tous les faits déformés et accrocs relatés dans les journaux durant la quinzaine précédente, les présentant comme une grave menace à l'identité québécoise, frisant le populisme en exacerbant les craintes et les tensions larvées dans la population.

Le chef de l'ADQ, Mario Dumont, put revenir à la charge durant les fêtes de Noël et du Nouvel An. Le 15 décembre, un employé du Patrimoine canadien, organisme fédéral qui gère le programme du multi-

$$
++
$$

13. Jean-Marc LÉger, "Une guerre de médias? ", La Presse, 18 janvier 2007, p. A21.

14. Benoît LeNOBle et Cyril LemieuX, "Oui, les médias font l'élection! ", htrp://medias.blog.lemonde.fr/ 2007/03/23/oti-les-medias-font-lelection/ (31 ju1ller 2007). 
culturalisme, dénonçait une invitation de cet organisme à célébrer le "solstice d'hiver" plutôt que la Noël'15. De plus, à l'Assemblée nationale, le souhait "Joyeuses fêtes" remplaçait de plus en plus le traditionnel "Joyeux Noël". Mario Dumont réclama un "accommodement raisonnable" pour pouvoir souhaiter à tous un "Joyeux Noël". Enfin, dès le début de la publication des résultats du sondage Léger Marketing, Dumont fit parâtrre, le 16 janvier 2007, une lettre ouverte dans laquelle il invitait la population québécoise à surmonter son "vieux réflexe de minoritaire» et à cesser de courber l'échine devant les "communautés culturelles", et proposait une constitution québécoise pour encadrer les accommodements raisonnables ${ }^{16}$. L'ADQ s'était imposée dans ce dossier et, en dépit d'un programme boiteux, elle fit élire 41 députés lors des élections du 26 mars 2007 . Ce résultat ouvre la perspective d'un gouvernement majoritaire pour le Parti conservateur du Canada, qui dirige actuellement un gouvernement minoritaire à Ottawa et dont les principaux gains au Québec lors des élections de 2006 furent réalisés en "territoire adéquiste".

Au cours des années, les instances de l'État ont manqué à leur responsabilité de clarifier la distinction entre accommodements raisonnables et arrangements à l'amiable, et de rappeler le socle de principes et de valeurs fondamentales du Québec. Pis encore, pendant la période électorale, plusieurs observateurs ont noté la passivité des partis politiques dans ce dossier. Le Parti québécois, craignant sans doute d'être taxé de racisme ou de xénophobie, avocat d'un nationalisme civique depuis les années 1990, négligea de se poser, à l'instar de l'ADQ, comme défenseur de l'identité ou des valeurs québécoises. Ce sera l'une des causes de la perte de son statut d'opposition officielle. Le Parti libéral au pouvoir essaya de calmer le jeu dans les moments les plus intenses, mais de façon plutôt tiède. Devant affronter l'électorat québécois avec un bilan fortement critiqué, il n'était peut-être pas mécontent de se retrouver dans une lutte à trois. Aux élections provinciales du 26 mars 2007, le Parti libéral perdit 28 sièges, passant de 76 à 48 élus, et se retrouva à la tête d'un gouvernement minoritaire.

$$
4 \div+
$$

15. Gérard CODERRE, a Un problème, fêter Noèl? », La Presse, 15 décembre 2007, hrtp://www.cybetpresse.ca/ article/20061214/CPOPINIONS/612140862/5288/CPOPINIONS (31 juillet 2007).

16. Mario DUMONT, "Une constitution québécoise pour encadrer les "accommodements raisonnables". Pour en finir avec le vieux réflexe de minoritairex, La vse rurale, 16 janvier 2007, http://www.la-vie-rurale.ca/contenu/10588 (31 juillet 2007). 


\section{UNE CONJONCTURE IDEOLOGIQUE CRUCIALE DANS LA SOCIETÉ QUEBECOISE}

Si cette question des accommodements raisonnables a pu revêtir une telle importance au cours de 2006-2007 et a mis l'accent sur les demandes des musulmans en particulier, nous estimons qu'elle renvoie à des enjeux sociétaux plus larges et à des facteurs internationaux qui ont exercé une influence structurante dans le débat québécois.

Au niveau international, un nouvel air du temps a caractérisé la décennie 1990 et les années 2000 : retour de la droite et du conservatisme, rappel à l'ordre public, injonction de cohésion sociale et de sécurité nationale. Le multiculturalisme, entendu au sens de projet de société (et non simplement de réalité démographique ou de politique publique), pose problème: on craint le communautarisme. Les pays occidentaux cherchent à revaloriser leur citoyenneté.

Mais surtout, les événements du 11 septembre 2001 ont provoqué un nouveau codage de l'ennemi extérieur et intérieur, le renforcement d'un discours dangereux et réducteur sur le choc des civilisations, des dichotomies simplistes: tradition/modernité, féodalisme/civilisation. Le dispositif discursif du néo-racisme qui se fonde sur le postulat de l'irréductibilité et de l'incompatibilité des cultures se décline maintenant sous diverses formes et affecte différentes cibles selon les contextes nationaux. Les enjeux sociétaux nationaux concernent, entre autres, la juridicisation du politique, la sécularisation de l'espace public et la question identitaire.

\section{LA JURIDICISATION DU POLITIQUE}

Depuis une vingtaine d'années, on observe dans plusieurs sociétés occidentales une tendance grandissante à recourir aux tribunaux pour statuer sur des questions sociales fondamentales ${ }^{17}$. L'une des conséquences de cette tendance a été d'amener des acteurs sociaux à ajouter à leur stratégie d'intervention dans l'espace public le recours direct aux tribunaux. La logique juridique contribue à modifier la manière dont les accommodements sont négociés, dans la mesure où les décisions judiciaires s'inscrivent dans une logique de "gagnants" et de "perdants", réduisant par le fait même l'espace de délibéra-

$$
++4
$$

17. Janet HIEBERT, Des droits à interpreter. Les juges, Le Parlement et l'elaboration des politiques publiques, Montréal, Institut de recherche en politiques publiques, juillet 1999, coll. "Choix. Tribunaux et législatures ; Marc CHEVRIER, "La démocratie représentative et la juridicisation du politıque ", communication présentée à l'ACFAS, 2004, http://agora.qc.ca/reftext.nsf/Documents/Politique. 
tion. Dans ce contexte, il n'est pas surprenant de constater que des groupes religieux intégristes utilisent aussi les tribunaux pour faire prévaloir leurs principes.

Ce phénomène a sans doute diverses causes, comme l'évolution importante du droit international, qui a contribué à définir des normes auxquelles les États nationaux doivent se conformer, mais aussi l'évolution du droit interne par l'adoption de chartes définissant et encadrant de manière plus ou moins serrée la façon dont les droits fondamentaux doivent être interprétés. Au Canada, cette transformation de la place du droit dans la société est liée à l'adoption de la Charte des droits et libertés et à son enchâssement dans la Constitution en 1982. Il est vrai que le Québec avait adopté dès 1975 sa propre charte des droits, un texte quasi constitutionnel qui va plus loin que la charte canadienne, s'appliquant non seulement aux lois et règlements mais également aux relations entre citoyens ou entre citoyens et institutions.

La question des rapports entre majorités et minorités est au cœur de la démocratie. Dans le cas des minorités issues de l'immigration, le Québec et le Canada ont adopté des idéologies et des politiques d'aménagement de la diversité (ou du pluralisme culturel) différentes: multiculturalisme à Ottawa, interculturalisme à Québec. Or, comme le souligne José Woehrling:

Les mécanismes modernes de protection des droits, qui reposent sur des instruments constitutionnels et sur l'intervention du pouvoir judiciaire, ont des effets surtout centralisateurs et uniformisateurs. En termes de centralisation, ils entraînent un transfert d'autorité des organes représentatifs locaux à des organes judiciaires fédéraux et contribuent à consolider la citoyenneté nationale au détriment de la citoyenneté locale ${ }^{18}$.

Cette question de la juridicisation des rapports sociaux, ressentie dans tout le Canada, renvoie également à la place du Québec dans la Confédération canadienne. Les décisions de la Cour suprême sur les accommodements raisonnables ont donc pu être perçues comme une imposition de la vision de l'idéologie du multiculturalisme du gouvernement fédéral ${ }^{19}$.

$$
+4
$$

18. José WOEHRLING, "Fédéralisme et protecrion des droits er libertés au Canada \#, communication présentée à l'Universicé Libre de Bruxelles, cycle de Conférences sur le fédéralisme comparé, 23 avril 2004, htrp:// www.ulb.ac.be/droit/droitpublic/docs/ woehrling.pdf (31 juillec 2007).

19. Hélène BuzETTl, «La Charte de l'exclusion?», Le Devoir, 10 avril 2007, p. Al-A8. 


\section{LA SECCULARISATION DE L'ESPACE PUBLIC}

Au cours des siècles, l'identité québécoise s'est construite autour d'une communauté historique et imaginée, d'un mythe fondateur - celui de la conquête de la Nouvelle-France et de la pérennité d'une société francophone en Amérique du Nord -, d'une langue et d'une foi, d'une pensée libérale et conservatrice en alternance, d'un projet nationalitaire - autonomie/ indépendance -, d'un gouvernement à compétence limitée au sein de la fédération canadienne, d'un modèle de solidarité sociale, et d'un socle de principes et de valeurs politiques. Or, parmi les valeurs politiques qui se sont consolidées au cours de la Révolution tranquille figure la laïcité ou le mouvement de sécularisation en cours, dont les modalités continuent de susciter des controverses.

Se pose alors la question du rapport à l'héritage religieux et de la place de la religion dans la cité. Le sujet oppose ceux qui veulent expurger les référents religieux de l'espace public au nom de normes universelles (qui sont souvent celles des majorités) et ceux qui soutiennent que les appartenances religieuses doivent avoir droit de cité, et qui défendent en conséquence des aménagements dans les institutions et les entreprises (par exemple, des banques de congés «neutres» et disponibles que les individus peuvent utiliser lors de fêtes religieuses, etc.).

Confinement strict du religieux dans l'espace privé? Admission dans l'espace public de la perpétuation d'un certain nombre de traditions, de symboles, de rites religieux? Cette deuxième option a été présentée tantôt comme un "accommodement raisonnable" au profit de la majorité (par exemple, le maintien du crucifix à l'Assemblée nationale), tantôt comme un "accommodement raisonnable" au profit des minorités soupçonnées de "vouloir imposer leurs us et coutumes et leur volonté à la majoritén.

En raison de l'absence de consensus sur le modèle de la laïcité, la discussion sur les accommodements raisonnables a conduit à un dérapage. L'autorisation de porter des signes religieux particuliers (le voile, le kirpan, etc.) dans des institutions publiques a donc été perçue par certains comme susceptible de retarder de façon décisive la sécularisation en cours. Mais la controverse autour de l'accommodement raisonnable s'articule également autour de la question identitaire. 


\section{QUESTION IDENTITAIRE ET NATIONALISME}

Revivifié par la question identitaire, un débat de plus en plus saillant sur le nationalisme ethnoculturel et le nationalisme civique a refait surface au cours des dernières années. Là encore, ce débat a contribué à orienter l'analyse du statut des accommodements raisonnables ${ }^{20}$.

Pour certains, la nation ethnoculturelle doit revêtir une connotation positive longtemps occultée ou reléguée au second plan : la revalorisation du passé des Canadiens français, groupe majoritaire d'ascendance et de culture française, concentré aujourd'hui sur le territoire du Québec. Selon eux, les tentatives d'articuler le débat en termes exclusivement civiques comme on l'a fait au Québec dans la période postréférendaire de 1995 contribuent à désincarner la nation en niant les origines historiques de la société québécoise et le rôle fondateur d'une majorité d'origine canadienne-française. Ainsi, on attribue au nationalisme civique une "trudeauisation" "qui considère les sociétés comme de vastes coalitions d'ayants droit liés par les chartes, non plus comme des communautés de culture et de mémoire ${ }^{21}$ ". En conséquence, "l'identité québécoise n'est pas respectée comme elle le devrait ${ }^{22}$ ", et certains attribuent à ce courant idéologique la dégringolade du Parti québécois et la disqualification relative dans l'opinion publique du projet souverainiste.

Un certain mouvement va même jusqu'à défendre l'affirmation des aspirations de la majorité d'origine canadienne-française sur la base d'un discours condamnant le "nettoyage civique de l'option souverainiste", la "tyrannie des minorités", le "reniement de soi", "l'orgie identitaire", la "balkanisation de la société", les "accommodements déraisonnables" et le multiculturalisme.

Or, la pensée sur la citoyenneté qui a influencé le nationalisme civique au Québec a fait l'objet de réflexions académiques et politiques bien avant le référendum sur la souveraineté de 1995. Ce courant d'idées s'appuie sur un vaste corpus d'études émergeant au cours des décennies 1980 et 1990 , qui portaient sur l'autonomisation ("empowerment»), la présence et la représentation des minorités sociologiques de toutes sortes dans les lieux du politique marqués par des rapports de pouvoir et des discriminations.

$$
4+
$$

20. Micheline LABELlE, "La "narion ethnique" et la nacion civique. Une fausse opposition n, Le Devoir, 5 février 2007, p. A7.

21. Éric BEDARD, op. cit.

22. lbud. 
Par ailleurs, la défense d'un pacte civique à conclure entre Québécois de toutes origines ne nie pas l'aspiration historique des Québécois d'origine canadienne-française. Cette pensée se base sur des acquis, entre autres en matière de politiques publiques. En effet, tous les gouvernements du Québec ont pris leurs distances avec la politique fédérale du multiculturalisme, et ce, depuis la décennie 1970. Cependant, tous ont adopté le pluralisme et la reconnaissance de la diversité comme principes et valeurs politiques centrales de la société québécoise, en lien avec la promotion d'un sentiment d'appartenance à la société québécoise.

Trop souvent, le débat masque la reconnaissance des acquis en ce domaine. Ces dispositifs se fondent sur les principes et les valeurs suivants: le français comme seule langue officielle, la démocratie, la résolution pacifique des conflits, les droits fondamentaux de la personne, la laïcité, le pluralisme, l'égalité des hommes et des femmes, la solidarité collective, le respect du patrimoine culturel (et/ou civique, selon les gouvernements), le respect des droits historiques de la minorité anglophone du Québec et des droits des Autochtones. Il est clair que ce socle de valeurs politiques prend son sens dans un contexte particulier: celui de l'histoire du Québec, celui de la société québécoise. Il n'est ni neutre ni abstrait.

Or, dans la question des accommodements raisonnables, ce qui a été perçu comme une menace porte essentiellement sur le fait que les signes religieux posent des défis particuliers relativement aux principes de l'égalité des hommes et des femmes et de la laïcité.

En dépit des manipulations et des dérapages que l'on a pu observer au cours de la période étudiée, la délibération sur les accommodements raisonnables garde toute sa légitimité. La consultation menée par la Commission de consultation sur les pratiques d'accommodements reliées aux différences culturelles, amorcée à l'automne 2007, fournira un cadre idéal pour l'expression de cette délibération collective. Certains enjeux préalables au débat sont d'ordre méthodologique: 1 ) dissocier la notion d'accommodement raisonnable (un droit balisé par les tribunaux pour mettre un terme à une discrimination qui porte atteinte à un droit inscrit dans les chartes canadienne et provinciales) des bonnes pratiques de gestion de la diversité au sens large observées dans les grandes entreprises privées et d'État et les institutions publiques; 2) dissocier l'accommodement raisonnable de la question de l'immigration, puisque la majorité des demandes d'accommodement concernent les personnes souffrant d'un handicap et les minorités religieuses de souche ancienne (protestants, témoins de Jéhovah, Juifs hassidiques, etc.); 3) dissocier les interrogations 
légitimes sur l'accommodement raisonnable des attitudes anti-immigration. À cet égard, il faut rappeler que, si, en $2006,58,6 \%$ des Québécois estimaient que la société est trop tolérante en matière d'accommodements, $68,1 \%$ d'entre eux considéraient que l'immigration est un atout pour la société.

Le débat sur les accommodements raisonnables fournit donc l'occasion de revisiter les représentations de l'identité québécoise, de son devenir, de ses valeurs politiques fondamentales, de sa spécificité. Le phénomène s'inscrit dans une tendance plus générale observable à l'échelle internationale. Dans ce contexte, il faut toutefois rappeler certains écueils et certaines menaces.

Un premier danger concerne la résurgence du racisme. Au cours de la dernière décennie, le climat néoconservateur et l'environnement géopolitique international ont exercé une influence significative et délétère sur l'opinion publique, influence qu'on ne peut minimiser. Le racisme est en augmentation dans toutes les sociétés occidentales, selon de nombreux rapports des Nations Unies.

Le renforcement des frontières territoriales observé depuis le 11 septembre 2001 a opéré parallèlement avec le renforcement des frontières identitaires. La question des religions a pris une place prépondérante dans les débats, et on assiste au renforcement de visions globalisantes des cultures et des religions. Ainsi, on a noté partout le retour en force des clichés orientalistes sur l'islamisation des sociétés, la soumission de la femme musulmane, la décadence des États gouvernés par l'islam, le terrorisme, etc.

Le discours néo-raciste et l'obsession sécuritaire contribuent à la production de discours menaçants sur un présumé choc des civilisations. À cet égard, la thèse de Samuel P. Huntington ${ }^{23}$, qui soutient que les conflits de civilisations domineront à l'avenir la "politique globale», semble avoir eu un certain effet performatif. On sait qu'elle repose sur le postulat selon lequel les différences culturelles et religieuses ne sont pas modifiables et que, en conséquence, les conflits culturels sont moins faciles à résoudre que les différences politiques et économiques. Or, cette thèse est réductrice, voire simpliste.

Cependant, il faut admettre que le retour du religieux s'accompagne d'expressions intégristes et fondamentalistes, avec en creux

$$
+4
$$

23. Samuel P. HuNTtNGTON, Le choc des civilusations, Paris, Odile Jacob, 1997. 
l'augmentation des inégalités sociales et de la racialisation de la pauvreté à l'échelle internationale et canadienne.

Enfin, les expressions du racisme n'ont pas atteint au Québec (et au Canada) le niveau du "racisme politique", qui est caractéristique des partis d'extrême droite européens. Cependant, à partir du moment où un parti politique, en pleine période électorale, utilise sciemment un thème comme l'accommodement raisonnable pour susciter une levée de boucliers autour de l'identité nationale, il y a lieu d'adopter une posture d'extrême vigilance. Les réflexions et les remises en cause autour de la question identitaire risquent de s'alimenter à un climat idéologique et à un air du temps qui ne portent plus l'ouverture des décennies antérieures.

Dans ce contexte, cette posture de vigilance concerne le Conseil de presse du Québec, dont le mandat consiste «à promouvoir le respect des plus hautes normes en matière d'éthique journalistique». S'il est exact que le Conseil "prend en compte l'évolution des valeurs et des mœurs de la société québécoise et canadienne ${ }^{24}$ ", il y a lieu d'appliquer cette réflexion aux moments critiques dont nous avons fait état dans ce texte.

Enfin, le rôle des représentants de l'État québécois est capital: il comprend le rappel constant et orchestré du socle de valeurs politiques fondamentales du Québec, voire l'élaboration d'une Constitution du Québec qui en précise les contours, ainsi que l'adoption et la mise en œuvre du projet de Politique gouvernementale de lutte contre le racisme et la discrimination.

$$
+4
$$

24. CONSEIL DE PRESSE DU QUÉBEC, «Droits ec responsabilités de la pressen, www.conseildepresse.qc.ca (25 avril 2007) 